\title{
Identification of TIMELESS and RORA as key clock molecules of non-small cell lung cancer and the comprehensive analysis
}

\author{
Haocheng Xian ${ }^{1}$, Yuan Li ${ }^{1}$, Boliang Zou' ${ }^{1}$ Yajuan Chen ${ }^{2}$, Houqing Yin ${ }^{1}$, Xuejun Li ${ }^{1}$ and Yan Pan ${ }^{1,3{ }^{*}}$
}

\begin{abstract}
Background: The incidence rate of non-small cell lung cancer (NSCLC) has been increasing worldwide, and the correlation of circadian rhythm disruption with a raised risk of cancer and worse prognosis has been shown by accumulating evidences recently. On the other hand, drug resistance and the impact of tumor heterogeneity have been inevitable in NSCLC therapy. These both lead to an urgent need to identify more useful prognostic and predictive markers for NSCLC diagnosis and treatment, especially on the aspect of circadian clock genes.

Methods: The expression of the main clock genes in cancer was probed with TIMER and Oncomine databases. The prognostic value of key clock genes was probed systematically with the Kaplan-Meier estimate and Cox regression on samples from TCGA database. RT-qPCR was performed on patient tissue samples to further validate the results from databases. The functional enrichment analysis was performed using the "ClusterProfiler" R package, and the correlation of key clock genes with tumor mutation burden, immune checkpoint, and immune infiltration levels were also assessed using multiple algorithms including TIDE, TIMER2.0, and XCELL.

Results: TIMELESS was significantly upregulated in lung tissue of clinical lung cancer patients as well as TCGA and Oncomine databases, while RORA was downregulated. Multivariate Cox regression analysis indicated that TIMELESS $(P=0.004, \mathrm{HR}=1.21[1.06,1.38])$ and $R O R A(P=0.047, \mathrm{HR}=0.868[0.755,0.998])$ has a significant correlation with overall survival in NSCLC. Genes related to TIMELESS were enriched in the cell cycle and immune system, and the function of RORA was mainly focused on oncogenic signaling pathways or glycosylation and protein activation. Also, TIMELESS was positively correlated with tumor mutation burden while RORA was negatively correlated with it. TIMELESS and RORA were also significantly correlated with immune checkpoint and immune infiltration levels in NSCLC. Additionally, TIMELESS showed a significant positive relationship with lipid metabolism.
\end{abstract}

Conclusions: TIMELESS and RORA were identified as key clock genes in NSCLC, and were independent prognostic factors for overall survival in NSCLC. The function of them were assessed in many aspects, indicating the strong potential of the two genes to serve as biomarkers for NSCLC progression and prognosis.

Keywords: Non-small cell lung cancer, Circadian clock genes, TIMELESS, RORA, Bioinformatics

*Correspondence: pannay26@bjmu.edu.cn

${ }^{3}$ Beijing Key Laboratory of Tumor Systems Biology, Peking University, Beijing 100191, China

Full list of author information is available at the end of the article

\begin{abstract}
Introduction
As the most common cancer type, lung cancer caused most cancer-related deaths worldwide, accounting for about 1.4 million deaths per year [1]. According to the cell origin, there are small-cell lung cancer and non-small cell lung cancer (NSCLC), in which $85 \%$ are
\end{abstract}

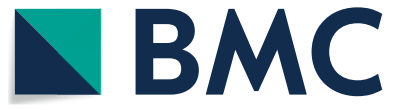
permits use, sharing, adaptation, distribution and reproduction in any medium or format, as long as you give appropriate credit to the original author(s) and the source, provide a link to the Creative Commons licence, and indicate if changes were made. The images or other third party material in this article are included in the article's Creative Commons licence, unless indicated otherwise in a credit line to the material. If material is not included in the article's Creative Commons licence and your intended use is not permitted by statutory regulation or exceeds the permitted use, you will need to obtain permission directly from the copyright holder. To view a copy of this licence, visit http://creativecommons.org/licenses/by/4.0/. The Creative Commons Public Domain Dedication waiver (http://creativeco mmons.org/publicdomain/zero/1.0/) applies to the data made available in this article, unless otherwise stated in a credit line to the data. 
NSCLC [2]. Although considerable efforts have been paid to elucidate its development and progression, the molecular mechanisms underlying NSCLC remain unclear [3]. Recently, compared with traditional chemotherapy, precision medicine, targeted therapy, and immunotherapy have led to an improvement of treatment outcomes in patients with NSCLC. However, drug resistance and the impact of tumor heterogeneity have been inevitable [4]. Therefore, the need to identify more useful prognostic and predictive markers or targets for the diagnosis and treatment of NSCLC is more and more urgent.

The circadian rhythm refers to the physiological and behavioral oscillations of an organism in a cycle of about $24 \mathrm{~h}$, behind which is a complicated system. The occurrence of circadian rhythm in mammals is based on endogenous clock genes, which mainly consist of Clock circadian regulator, brain and muscle ARNTlike 1, period circadian regulator 1 (PER1), PER2, cryptochrome circadian regulator 1 (CRY1), CRY2, timeless circadian regulator (TIMELESS), retinoic acidrelated orphan receptor A $(R O R A)$, nuclear receptor subfamily 1 , group D member 1 (NR1D1), and NR1D2 [5]. They synchronize multiple molecular, biochemical, physiological, and behavioral process, also, at the cellular level, lots of genes were regulated in expression or function transcriptionally or post-transcriptionally by these clock genes [6].

Circadian disruption is one of the group 2A human carcinogens identified by WHO [7]. Many epidemiological studies have shown that disorder of the circadian rhythm is related to worse prognosis and increased risks of cancer. For example, long-period shiftwork is correlated with higher risk of the development of prostate and breast cancers [8]. Also, the hazard ratio of rectal cancer [9] and lung cancer [10] is increased after exposure to shiftwork. Some in vivo experiments have also confirmed the pattern: hypothalamic suprachiasmatic nucleus ablation in normal mice or tumor-bearing mice strongly accelerates tumor progression [11, 12]. In addition, mice with genetic alterations in some clock genes can more easily develop teratomas, lymphoma, liver cancer, and ovarian cancer [13-15]. However, to date, the detailed effects of clock genes on NSCLC as well as their underlying mechanisms remain unclear.

As technology has developed, bioinformatics has been widely applied to genomic and proteomic studies and has provided an unparalleled scale to explore the correlation and interaction of molecules and many biological processes. In this study, using bioinformatics analysis, we identified TIMELESS and RORA as the key clock genes in NSCLC and showed their strong potential as key molecules underlying NSCLC progression and prognosis.

\section{Materials and methods}

\section{Datasets and data availability}

The sex, age, survival, status, topography and tumornode-metastasis (TNM) stage of lung adenocarcinoma (LUAD) and lung squamous cell carcinoma (LUSC) samples were extracted from The Cancer Genome Atlas (TCGA: https://cancergenome.nih.gov) with the exclusion patients without sufficient data were excluded from subsequent analyses. In total, 513 TCGA-LUAD samples and 501 TCGA-LUSC samples were qualified and used for subsequent analyses. Data from normal lung tissue samples were also obtained in GenotypeTissue Expression (GTEx) (https://gtexportal.org/ home/datasets).

\section{Clinical patient tissue sample collection}

After the approval of the ethics committees of the Third Affiliated Hospital of Kunming Medical University (No: KYCS202180), paired tumor tissue and adjacent normal tissue were obtained in Yunnan Cancer Hospital from 13 patients who was diagnosed with lung cancer. Written informed consent was obtained from all patients before the tissue sample collection.

\section{Oncomine database analyses}

As a comprehensive bioinformatics database, the Oncomine database (https://www.oncomine.org/) is established for providing and assessing cancer transcriptome data [16]. In this study, we compared the transcription levels of circadian genes between tumor specimens and normal tissue. The threshold value was set as follows: fold change more than $1.5, P$ value less than 0.001 , and gene ranking in top $10 \%$.

\section{Kaplan-Meier plotter database assay}

The Kaplan-Meier plotter (KM-plotter, http://kmplot. com/analysis/) includes 10,461 samples of cancer patients and can probe the effects of multiple genes on survival [17]. In our study, this database was used to analyze correlation of TIMELESS, RORA, PER1, PER2, and $C R Y 2$ expression with survival in lung cancer. The $\log$-rank $P$ value were calculated as well as the hazard ratio (HR) with $95 \%$ confidence intervals (CIs).

\section{HR and overall survival nomogram model}

To identify the proper terms for the nomogram, we did univariate and multivariate Cox regression analyses. The $P$ value, HR, and $95 \% \mathrm{CI}$ of each variable were plotted in the forest plot. And a nomogram model based on the multivariate Cox regression model was developed as a visual representation to predict the recurrence 
risk of 1, 2, 3, and 5 years, respectively. "Forestplot" and "rms" of R software package 4.0.3 were used.

\section{RT-qPCR}

Total RNA was extracted from clinical samples using TRIzol reagent (Invitrogen, California, USA) according to the manufacture's protocol, and the cDNA was synthesized using the cDNA First Strand Synthesis Kit (Abclonal, WuHan, China). RT-qPCR was performed according to the instructions of SYBR Green Fast qPCR Mix (Mei5bio, Beijing, China) with following conditions: $95^{\circ} \mathrm{C}$ for $30 \mathrm{~s}$, then 45 cycles including $95^{\circ} \mathrm{C}$ for $5 \mathrm{~s}$ and $60^{\circ} \mathrm{C}$ for $30 \mathrm{~s}$ for annealing and elongation. The relative expression of target genes was normalized to GAPDH and quantified with $2^{-\Delta \Delta \mathrm{Ct}}$ method [18]. The oligonucleotide primer sequences used were as follows: TIMELESS forward 5'- GTTTTGGCAATCTGCCTAAGGA-3', and reverse 5'- GCAGCTCATACAAGGTTTCACT-3'; RORA forward 5' - CACGACGACCTCAGTAACTACA$3^{\prime}$, and reverse 5' - TGGTGAACGAACAGTAGGGAA3'; GAPDH, forward 5'- GAGTCCACTGGCGTCTTC AC- $3^{\prime}$, and reverse $5^{\prime}$ - TGGTTCACACCCATGACG AA $-3^{\prime}$.

\section{Identification of genes related to TIMELESS and RORA in NSCLC samples}

The "Limma" R package was applied to assess the mRNA expression of samples with higher and lower TIMELESS and RORA expression divided according to the median. The threshold was defined as adjusted $P$ value $(\mathrm{FDR}<0.05)$ and the fold change (more than 1.5). The package version used in the present study is 3.18.0.

\section{Functional enrichment analyses}

The "ClusterProfiler" $\mathrm{R}$ package was used to perform Gene Ontology (GO) and Kyoto Encyclopedia of Genes and Genomes (KEGG) pathway enrichment analyses with those whole genomic genes as enrichment background [19]. Terms with a $P$-value $<0.01$ and an enrichment factor $>1.5$ were grouped into clusters based on the membership similarities. The detailed method was as described previously [20].

\section{Analyses of tumor mutation burden and correlation with gene expression levels}

The correlation of TIMELESS and RORA expression from TCGA database with tumor mutation burden were analyzed by Spearman's correlation analysis. And the results were visualized with the "ggplot2" package (tumor mutation burden [TMB] score and genes) and "pheatmap" package (gene correlations) of $\mathrm{R}$ software 4.0.3. $P<0.05$ has statistical significance.

\section{Prediction of immune checkpoint blockade response}

The tumor immune dysfunction and exclusion (TIDE) algorithm was mainly used to predict the immune checkpoint blockade (ICB) response as previously described [21]. Multiple gene expression markers were used to estimate the dysfunction and exclusion of tumor infiltration cytotoxic T lymphocytes (CTLs).

\section{Analysis of immune infiltration level}

As a comprehensive database, TIMER2.0 (http://timer. comp-genomics.org/) could predict and estimate the immune infiltration levels by multiple immune deconvolution methods [22]. In this study, it was used to explore the correlations of immune cell infiltration levels $(\mathrm{CD} 8+\mathrm{T}$ cells, CD4 $+\mathrm{T}$ cells, B cells, myeloid dendritic cell $[\mathrm{mDCs}]$, neutrophils, and macrophages) with TIMELESS and RORA expression adjusted by tumor purity. The correlation of immune infiltration levels with TIMELESS and RORA expression levels was also analyzed by the "immuneeconv" $\mathrm{R}$ package. The data were visualized using the "ggplot2" package and "pheatmap" package. Spearman's correlation analysis was used and $P<0.05$ was considered to have statistical significance.

\section{The mutation situation analysis}

cBioportal (cbioportal.org) is a comprehensive tool for multidimensionally assessing cancer data from the level of genomics [23]. In this study, the cBioportal database was used to examine the mutation situation of TIMELESS and RORA in NSCLC samples, and lollipop plots were plotted for each gene.

\section{Statistical analysis}

Data are shown as the mean $\pm \mathrm{SD}$. The correlation was evaluated by Spearman's correlation analysis. Survival curves were generated by the Kaplan-Meier method with the log-rank test. Student's $t$-test was used to compare the difference within two groups, and the KruskalWallis test was used to compare the difference among more than two groups unless otherwise stated. $P<0.05$ was considered statistically significant.

\section{Results}

The mRNA levels of five clock genes (TIMELESS, RORA, $P E R 1, P E R 2, C R Y 2)$ are significantly altered in several kinds of cancer

The transcription level of clock genes in tumor and normal tissues was assessed with Oncomine database (Fig. 1A). we found that the TIMELESS expression level was upregulated in most of the significantly altered datasets (77 of 84 datasets, $91.7 \%$ ), especially in breast 
cancer and lung cancer, whereas the expression levels of RORA, PER1, PER2, and CRY2 were downregulated in most of the significantly altered datasets (63 of 73 datasets, $86.3 \%$; 44 of 49 datasets, $89.8 \%$; 44 of 48 datasets, $91.7 \%$; 49 of 52 datasets, $94.2 \%$ ).

To further confirm the alterations in transcription level of these five clock genes, we repeated the mRNA levels detection of TIMELESS, RORA, PER1, PER2, and CRY2 using the TCGA RNA-seq data (Fig. 1B). TIMELESS expression was upregulated significantly in most cancer types $(P<0.05)$, while it was downregulated in kidney cancer (KICH and KIRC). RORA expression was significantly lower in multiple cancer types than that in corresponding normal tissues $(P<0.05)$, while it was upregulated in KIRC.

\section{TIMELESS and RORA were identified as key clock genes in NSCLC}

As TIMELESS expression was significantly upregulated and RORA, PER1, PER2, and CRY2 expression was significantly downregulated in most cancer types including LUAD and LUSC, it is necessary to probe their prognostic values (Fig. 2A-E). The log-rank test indicated a significant association of mRNA levels with overall survival (OS) of lung cancer patients $(P<0.05)$, and the TIMELESS, RORA, PER2, and CRY2 expressions were significantly correlated with progression-free survival (PFS) in lung cancer patients $(P<0.05)$. The TIMELESS and RORA expression levels were also significantly correlated with post-progression survival (PPS; $P<0.05$ ). Collectively, lung cancer patients with high expression of TIMELESS or low expression of RORA, PER1, PER2, or CRY2 had a significantly poorer prognosis in OS.

To further probe and validate the prognostic value of these five clock genes, we used univariate and multivariate cox regression analyses and established a nomogram model to predict the OS of NSCLC patients (Fig. 3A-D). Univariate Cox regression analysis revealed that among the five clock genes, TIMELESS $(P=0.034, \mathrm{HR}=1.19$ $[1.09,1.32])$ and $R O R A(P=0.042, \mathrm{HR}=0.88[0.78,0.99])$ were correlated greatly with the OS of NSCLC (Fig. 3A). Moreover, multivariate Cox regression analysis indicated that TIMELESS $(P=0.004, \mathrm{HR}=1.21[1.06,1.38])$ and RORA $(P=0.047, \mathrm{HR}=0.868[0.755,0.998])$ were independent prognostic factors for OS in NSCLC (Fig. 3B). Based on that, an OS nomogram model was established to estimate the overall survival of NSCLC patients with TIMELESS and RORA expression levels, and age (Fig. 3C). The points of RORA, TIMELESS, and age were added to obtain the total points, by which the OS rate in $1,2,3$, and 5 years was predicted. The calibration curve (Fig. 3D) for the OS nomogram indicated a conceivable prediction effect of the model. These results indicated that TIMELESS and RORA had a high prognostic value in NSCLC.

We further explored the expression profiles of TIMELESS and RORA in different pathological TNM (pTNM) stages in NSCLC using data from TCGA database (Fig. 3E-F). TIMELESS expression in stage II NSCLC was much higher than that in stage I $(P<0.0001)$, while compared with stage I, RORA expression in stage III was significantly lower $(P<0.0001)$. Thirteen pairs of clinical NSCLC samples from the Third Affiliated Hospital of Kunming Medical University were used to confirm the expression pattern of TIMELESS and RORA using RTqPCR methods. TIMELESS was significantly upregulated (Fig. 3G, P < 0.05) while RORA was significantly downregulated (Fig. $3 \mathrm{H}, \mathrm{P}<0.0001$ ) in tumor tissue compared to the adjacent normal tissue.

\section{Genes and function related to TIMELESS and RORA in NSCLC TCGA samples}

To investigate the biological function of TIMELESS and $R O R A$, we used Limma $\mathrm{R}$ package to identify related genes of TIMELESS and RORA. The median of TIMELESS or RORA expression level was the basis by which the TCGA NSCLC samples were divided into two groups with a threshold of fold change $>1.5$ and $P<0.05$. Collectively, 1955 genes were significantly upregulated in the high TIMELESS expression group, indicating a significantly positive correlation with TIMELESS, whereas 799 genes were significantly negatively correlated with TIMELESS (Fig. 4A-B). Regarding RORA, 709 genes had significantly positive correlation, and 117 genes had a significantly negative correlation (Fig. 4C-D). The correlated genes are listed in Table S1.

GO and KEGG signaling pathway enrichment analyses were done for the upregulated or downregulated genes of TIMELESS (Fig. 5A1-A4) or RORA (Fig. 5B1-B4), respectively. Genes positively correlated with TIMELESS were mainly enriched in the cell cycle, and DNA replication. There are "mismatch repair" and "nucleotide excision repair" clusters in KEGG pathways and all clusters in $\mathrm{GO}$, indicating the strong impact of TIMELESS on the

(See figure on next page.)

Fig. 1 The mRNA expression level of clock genes in different cancers. A Datasets in which the mRNA expression levels of clock genes were significantly changed compared with normal tissues in Oncomine database. Color red represents upregulated genes while blue represents downregulated genes. The depth of the cell color represents the rank of target genes in all the significantly changed genes in a dataset. The threshold was set as: $P$-value of 0.001 , fold change of 1.5, and gene ranking of top 10\% $\mathbf{B}$ Expression levels of TIMELESS, RORA, PER1, PER2, and CRY2 in different cancer types from TCGA database were analyzed by TIMER. ${ }^{*} P<0.05,{ }^{*} P<0.01,{ }^{* * *} P<0.001$ 

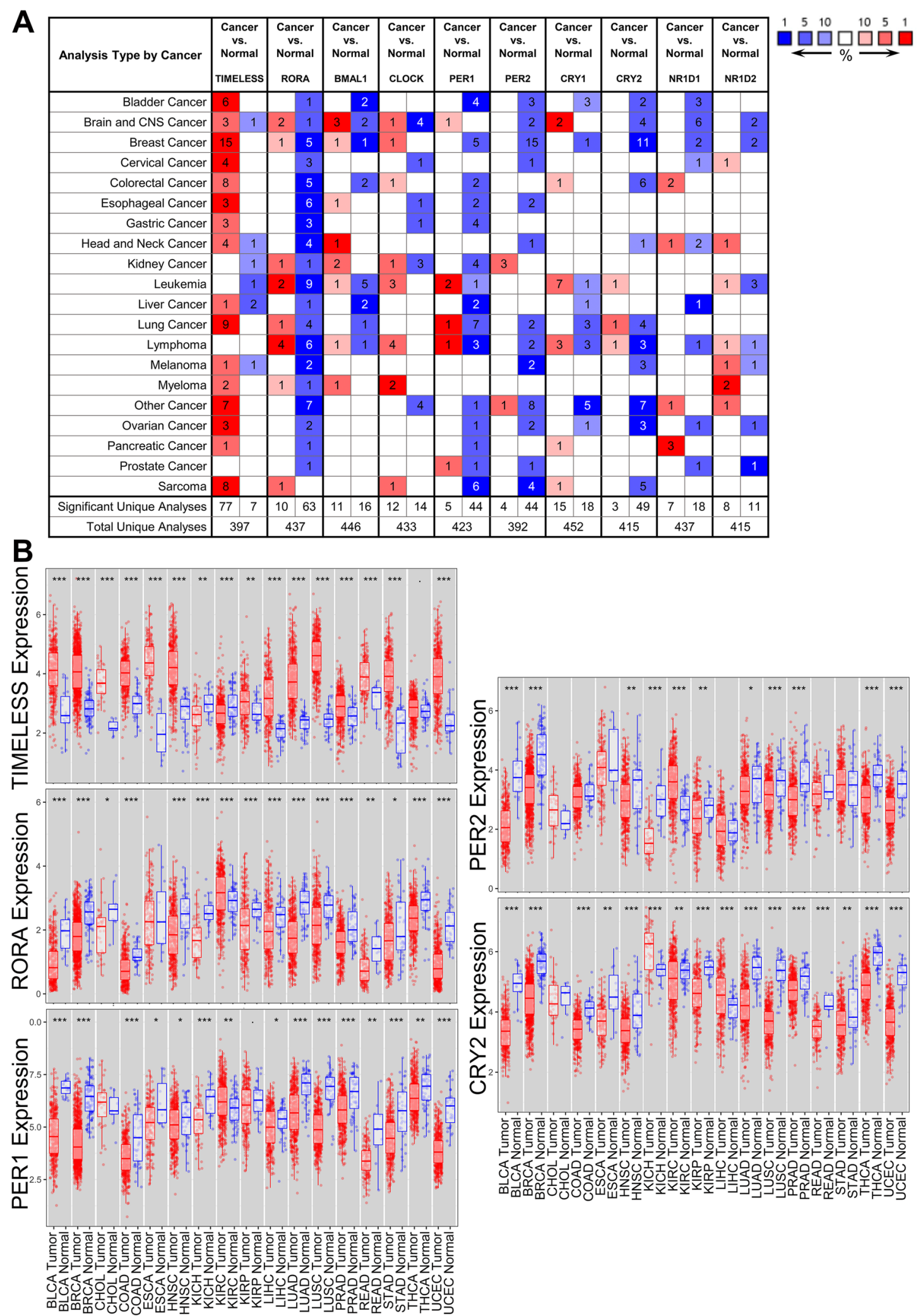

Fig. 1 (See legend on previous page.) 

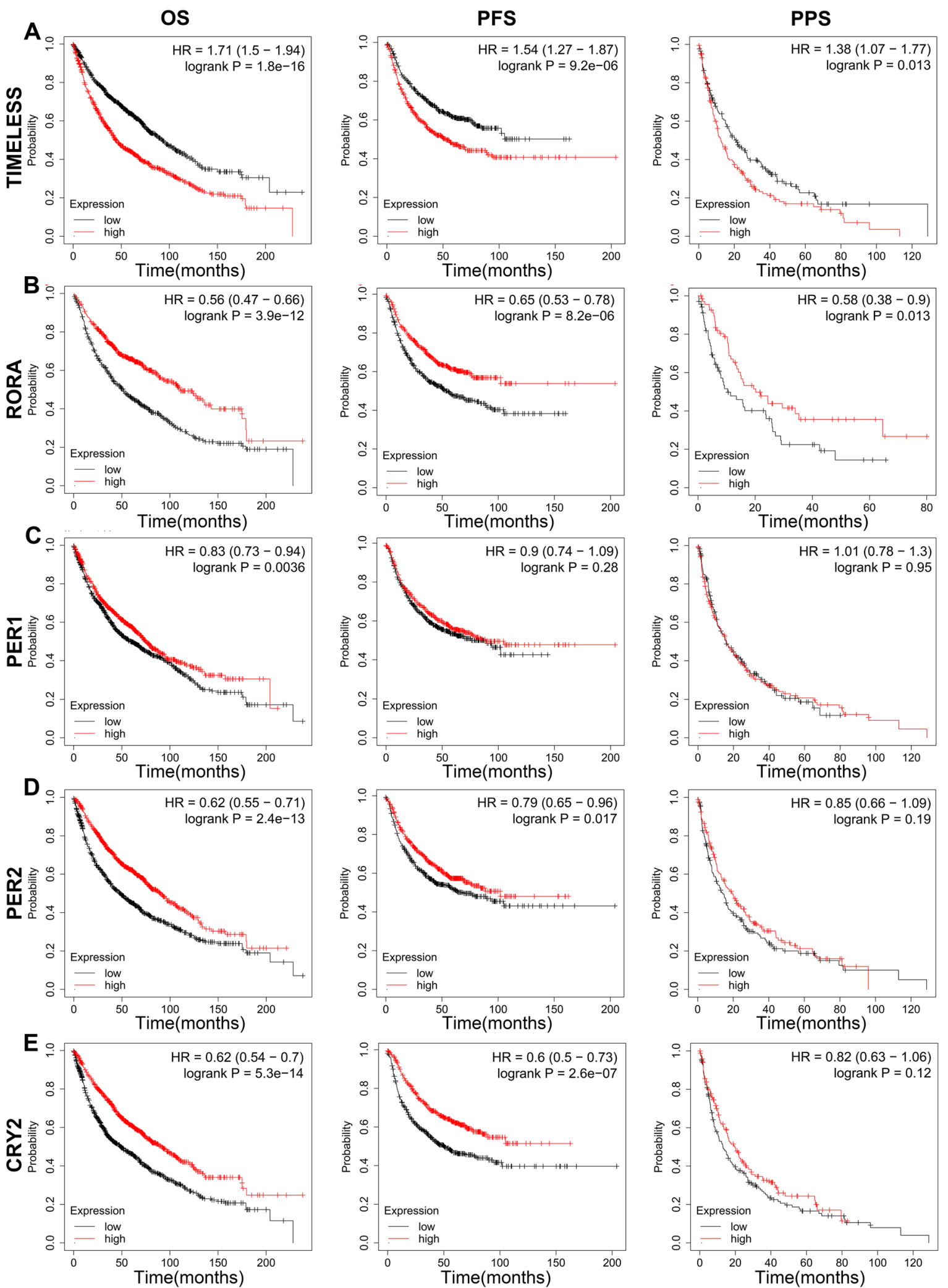

Fig. 2 Kaplan-Meier survival curve of clock genes. The correlations of OS, PFS, and PPS with TIMELESS (A), RORA (B), PER1 (C), PER2 (D), and CRY2 (E) expression in lung cancer were probed using KM plotter database. The hazard ratio (HR) and log-rank $P$-value were also shown on each curve 


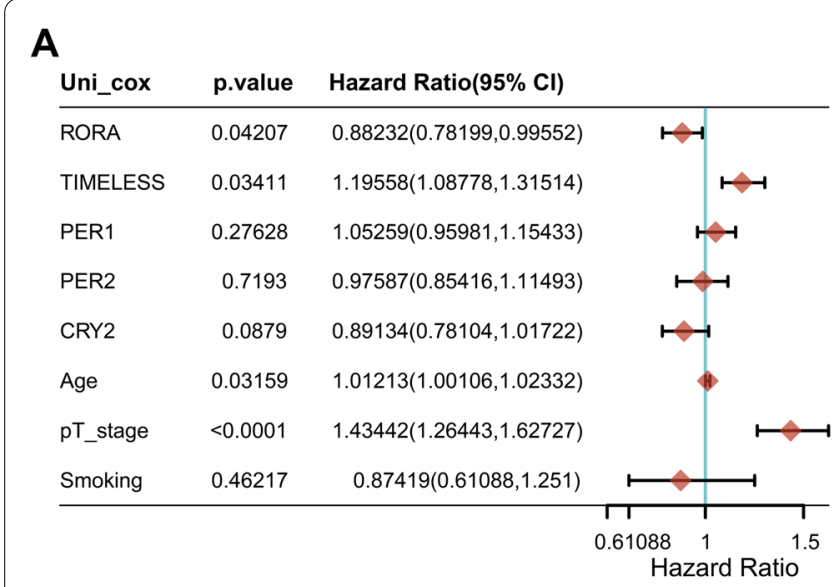

B

\begin{tabular}{|c|c|c|c|c|}
\hline Mult_cox & p.value & Hazard Ratio(95\% Cl) & & \\
\hline RORA & 0.04712 & $0.86833(0.75535,0.99821)$ & $\mapsto-1$ & \\
\hline TIMELESS & 0.00366 & $1.21073(1.06422,1.37741)$ & & $\longmapsto$ \\
\hline PER1 & 0.13126 & $1.12953(0.93406,1.30588)$ & & $\leadsto$ \\
\hline PER2 & 0.80852 & $0.9789(0.82381,1.16318)$ & & $\neg$ \\
\hline CRY2 & 0.24048 & $0.88627(0.72447,1.08422)$ & $\mapsto$ & -1 \\
\hline Age & 0.00517 & $1.01762(1.00524,1.03015)$ & & 4 \\
\hline Race & 0.56923 & $0.9207(0.69272,1.2237)$ & $\longmapsto$ & -1 \\
\hline pT_stage & $<0.0001$ & $1.4601(1.25029,1.70511)$ & & $\longmapsto$ \\
\hline Smoking & 0.59635 & $0.89355(0.58917,1.35519)$ & $\longmapsto$ & $\longrightarrow$ \\
\hline
\end{tabular}
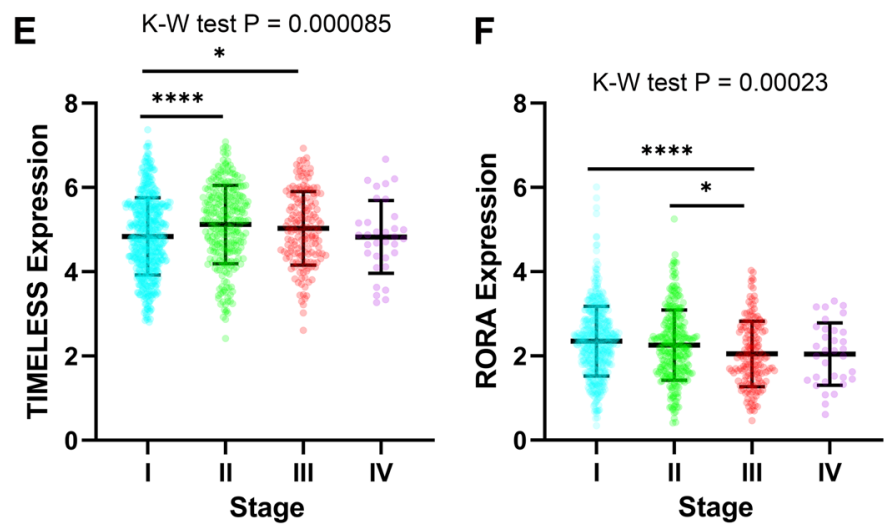

D
C

Points

RORA

TIMELESS

Age

Total Points

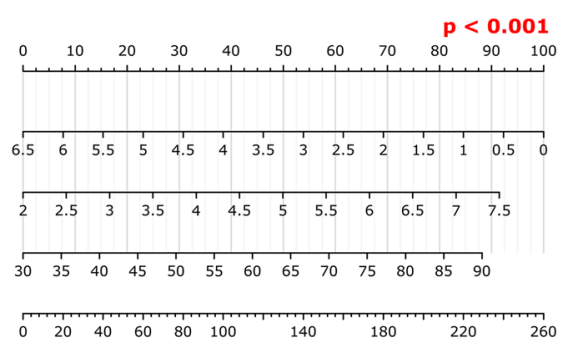

Linear Predictor

1-year survival Pro

2-year survival Pro

3-year survival Pro

5-year survival Pro

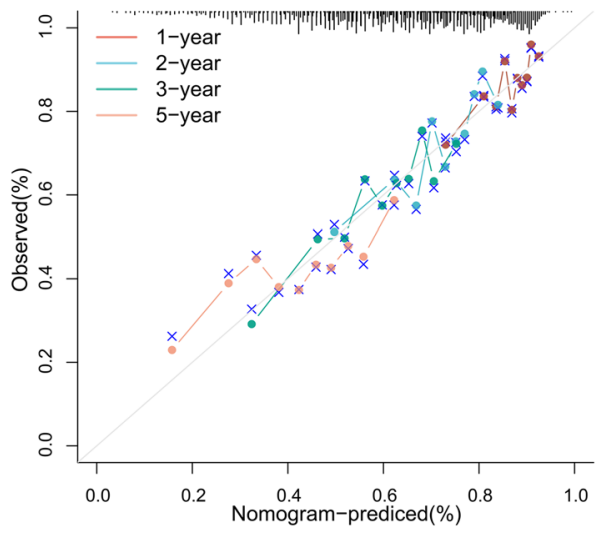

G

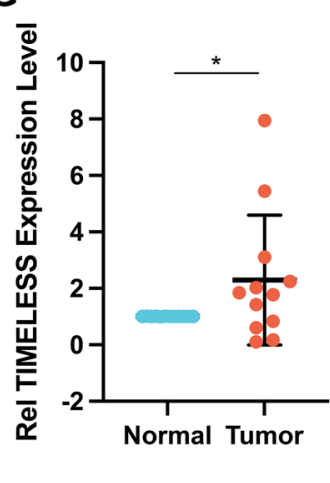

H

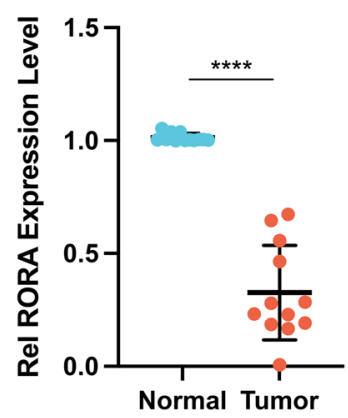

Fig. 3 Prognostic value of TIMELESS, RORA, PER1, PER2, and CRY2. The univariate (A) and multivariate cox regression analysis (B) of TIMELESS, RORA, PER1, PER2, and CRY2 levels as well as some clinical parameters including age, race, pathological tumor stage, and smoking status with overall survival in NSCLC samples from TCGA. C The nomogram to predict the 1,2,3, and 5-year overall survival of NSCLC patients by adding up the points of RORA, TIMELESS, age to the total points. D The calibration curve for the overall survival nomogram model. A dashed diagonal line represents the ideal nomogram, and four colored lines represent the 1,2,3, and 5-year observed nomograms. E-F Correlation of TIMELESS (E) and RORA (F) expression with pTNM stage of NSCLC in TCGA database. G-H TIMELESS $(\mathbf{G})$ and RORA (H) expression levels of tumor and adjacent normal tissues in NSCLC patients. ${ }^{*} P<0.05,{ }^{* * *} P<0.0001$ 

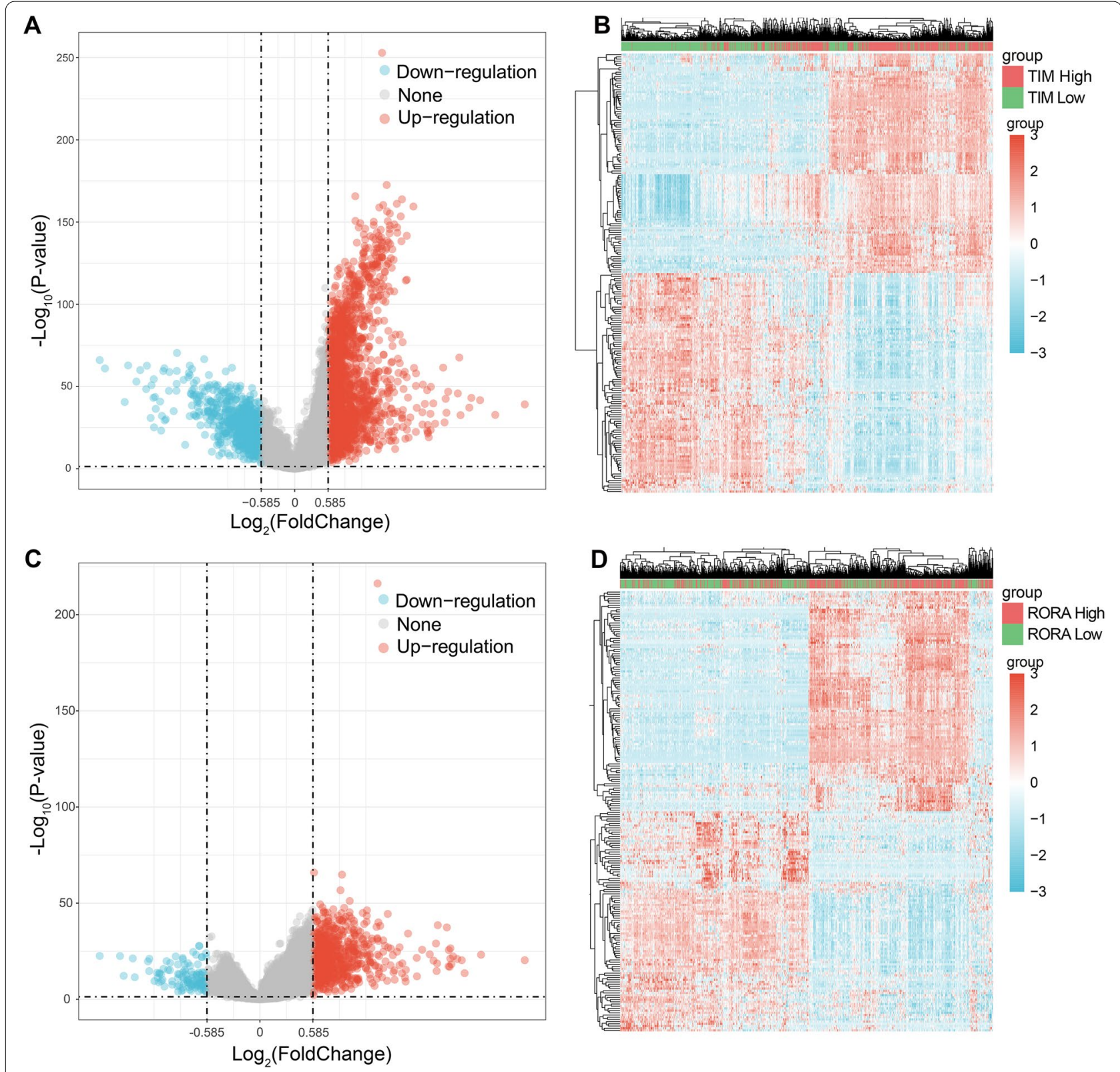

Fig. 4 Identification of genes corelated to TIMELESS and RORA in NSCLC samples. Volcano plots were constructed using fold change and adjusted $P$-value to probe the genes significantly correlated to TIMELESS (A) and RORA (C) expression level. The red point in the plot represents the over-expressed mRNAs and the blue point indicates the down-expressed mRNAs with statistical significance. The hierarchical clustering analysis of mRNAs, which were significantly differentially expressed between high and low expression of TIMELESS (B) as well as RORA (D). Fold change > 1.5 and $P<0.05$ were considered statistically significant

cell cycle (Fig. 5A1-A2). Interestingly, the significantly downregulated genes were mainly enriched in the clusters strongly related to the immune system; for example, antigen processing and presentation, complement and coagulation cascades, and cytokine-cytokine receptor interaction were directly related to immunological reactions, while other KEGG clusters were related to hypersensitivity or autoimmune diseases (asthma, systemic lupus erythematosus, and inflammatory bowel disease so on; Fig. 5A3 and A4). These results indicate that TIMELESS might exert cancer-promoting effects by influencing multiple immunologic processes.

Regarding RORA, the upregulated clusters in KEGG pathways were complicated, including many cancers and signaling pathways (e.g., Wnt signaling pathway, PI3K-Akt signaling pathway, Hedgehog signaling 


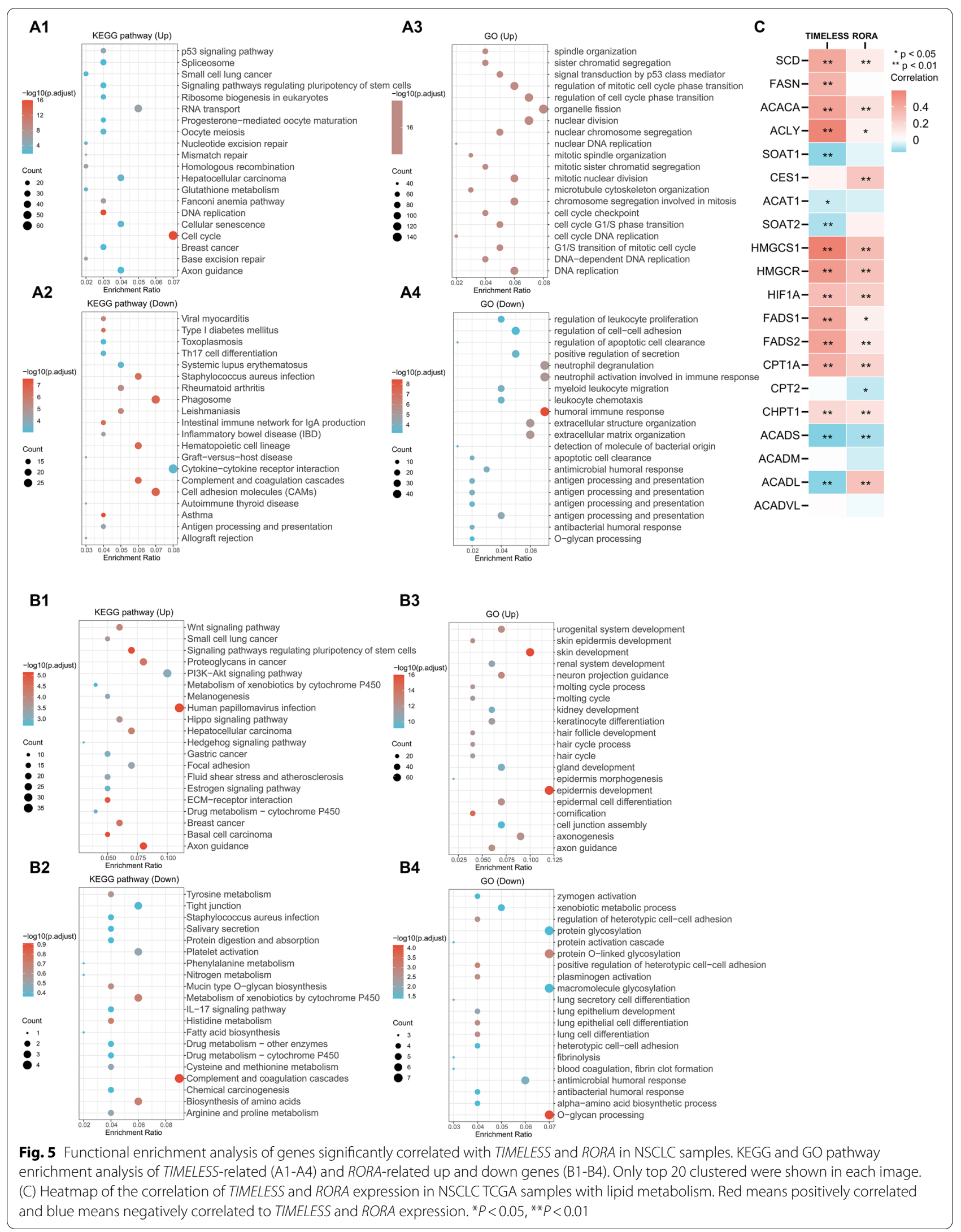


pathway; Fig. 5B1), while the upregulated GO clusters were enriched in embryonic development. The downregulated KEGG pathway was unenriched $(P>0.05)$, and the downregulated GO clusters were mainly enriched in glycosylation and protein activation (e.g., macromolecule glycosylation, protein glycosylation, and protein activation cascade). Interestingly, some clusters were related to lung cell development and differentiation, which might indicate the tissue specificity of the effect of RORA.

The correlation of TIMELESS and RORA expression with lipid metabolism is shown in Fig. 5C. Many important molecules in lipid metabolism had a significant correlation with TIMELESS and RORA expression level $(P<0.05)$. For example, SCD, FASN, ACACA, ACLY, HMGCS1, HMGCR, HIF1A, FADS1, FADS2, CPT1A, and $C H P T 1$ were significantly positively correlated with TIMELESS expression, and SOAT1, ACAT1, SOAT2, $A C A D S, A C A D L$ had a significantly negative correlation $(P<0.05)$.

\section{TIMELESS and RORA expression levels are associated with immune infiltration level in NSCLC}

As an independent predictor of the status of sentinel lymph node, tumor infiltrating lymphocytes have been gathering more and more attention; therefore, the probable correlation between TIMELESS and RORA expression and immune infiltration levels in NSCLC was assessed. We found that higher expression of TIMELESS led to the significant downregulation of B cells, CD4+ T cells, CD8+ T cells, neutrophils, macrophages, and $\mathrm{mDC}$ infiltration levels $(P<0.05$; Fig. 6A, C). Whereas, $R O R A$ expression was positively correlated with all six types of immune cells in NSCLC $(P<0.05$; Fig. 6B-C).

The results were further validated using the immunedeconv package of $\mathrm{R}$ software. Consistently, TIMELESS expression was also significantly negatively correlated with the infiltration of CD4 $+\mathrm{T}$ cells, $\mathrm{mDCs}$, and $\mathrm{B}$ cells in LUAD, and CD8+ T cells, neutrophils, macrophages, and $\mathrm{mDCs}$ in LUSC $(P<0.05$; Fig. 6D). Moreover, in LUAD, RORA expression was also significantly positively correlated with all six types of immune cells $(P<0.05$; Fig. 6E), which was in good agreement with the results using the TIMER algorithm.

To further assess the correlation, we examined the detailed infiltration level using the algorithm of XCELL database. The results showed that TIMELESS expression level was negatively correlated with most immune cell infiltration in both LUAD and LUSC $(P<0.05$; Fig. 7A). By contrast, the RORA expression level was positively correlated with most immune cell infiltration in LUAD $(P<0.05$; Fig. $7 \mathrm{~B})$, with no obvious correlation in LUSC.
TIMELESS and RORA are associated with TMB and immune checkpoint-related genes

To further assess the influence of TIMELESS and RORA on the effect of immune checkpoint inhibitors, the TMB was investigated. There was a significant positive correlation between TIMELESS expression and the TMB score in NSCLC $(P<0.0001, \rho=0.31$ [0.25, 0.37]; Fig. 8A). Also, the TMB score was negatively correlated with $R O R A$ expression in NSCLC $(P<0.0001, \rho=-0.18[-0.24$, -0.12 ]; Fig. 8B).

We also examined the association of TIMELESS and RORA with immune checkpoint-related genes (Fig. 8C). TIMELESS expression was positively correlated with CD274, LAG3, and PDCD1 in LUAD as well as CD274 in LUSC significantly $(P<0.05)$, and was negatively related to CTLA4 and HAVCR2 in LUSC $(P<0.05)$. RORA was significantly positively correlated with those immune checkpoint-related genes (CD274, CTLA4, HAVCR2, LAG3, PDCD1, PDCD1LG2, SIGLEC15, and TIGIT) in LUAD as well as with $C D 274$ and $P D C D 1 L G 2$ in LUSC $(P<0.05)$.

ICB therapy of cancer can bring various benefits. The response to this treatment of NSCLC patients with different TIMELESS or RORA expression were predicted by TIDE algorithm (Fig. 8D-G). In LUAD, the high expression of TIMELESS was correlated with a significantly higher TIDE score compared to patients with lower TIMELESS expression (Fig. 8D).

\section{The mutation of TIMELESS and RORA and its influence on immune cell infiltration in NSCLC}

We analyzed the mutation situation of TIMELESS and RORA in NSCLC. The whole mutation rates of TIMELESS and RORA were 2.8 and $1.2 \%$ in NSCLC samples with cBioportal database, respectively (Fig. 9A), and the gene mutation type detected in TIMELESS included missense and nonsense mutations, while in $R O R A$, the detected mutation consisted of only missense mutations (Fig. 9A-B). The detailed alteration status of the two clock genes is shown in Fig. 9C. Regarding TIMELESS, the alteration rate was higher in LUAD (3.1\% of 2583 cases) than in LUSC ( $1.77 \%$ of 1087 cases). In LUAD, the two major alteration types were amplification (1.94\%) and mutation $(1.12 \%)$, while in LUSC, the amplification rate was quite rare $(0.06 \%)$ and mutation was the major alteration type $(1.72 \%)$. Regarding RORA, the alteration rate was lower (1.39\% in LUAD and $0.89 \%$ in LUSC). Also, the dominant alteration type was mutation $(0.74 \%)$ in LUAD, while in LUSC, the deep deletion rate $(0.39 \%)$ was the same as the mutation rate.

Then the correlation between copy-number-change and the immune cell infiltration levels was analyzed 


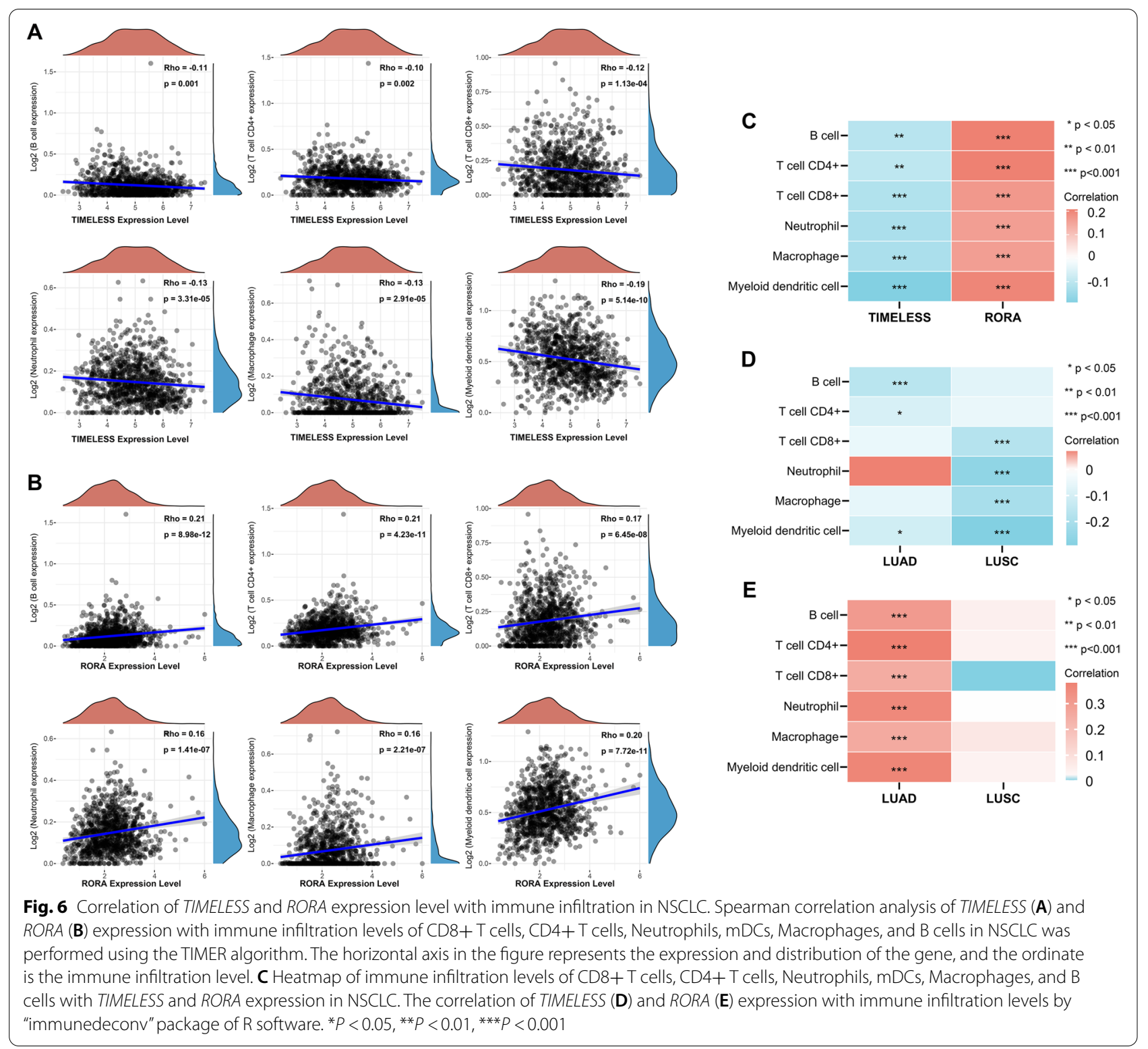

using the TIMER database (Fig. 9D). The arm-level gain of TIMELESS in LUAD and LUSC was in a significant correlation with most immune cell infiltration $(P<0.05)$. Regarding RORA, a significant negative correlation of deep deletion and arm level-gain with immune infiltration level was observed $(P<0.05)$.

\section{Discussion}

This study identified two important clock genes, TIMELESS and RORA, which were shown to have some relationship with NSCLC development and progression using bioinformatics analysis. TIMELESS mRNA and protein expression were upregulated, while the RORA expression were downregulated in NSCLC tissues. NSCLC patients with higher expression of TIMELESS or lower expression of RORA had a significantly poorer prognosis. The importance of the two genes in NSCLC was probed furtherly. Moreover, TIMELESS and RORA expression levels were two independent prognostic factors for OS in NSCLC. Also, we observed that TIMELESS expression in stage II NSCLC was higher than those in other stages and RORA expression in stage III was significantly lower compared to those at stage II or I.

Our findings with many independent datasets including thousands of samples are consistent with previous studies. Yoshida et al. found high TIMELESS expression 


\begin{tabular}{|c|c|c|c|c|c|c|c|}
\hline \multirow{2}{*}{ A } & \multicolumn{2}{|c|}{ TIMELESS } & & B & \multicolumn{2}{|c|}{ RORA } & \\
\hline & 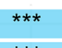 & 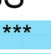 & \multirow{2}{*}{${ }^{*} p<0.05$} & \multirow{2}{*}{$\begin{array}{l}\text { stroma score } \\
\text { microenvironment score }\end{array}$} & **** & & \\
\hline microenvironment score & *** & $* * *$ & & & & & \multirow{2}{*}{$\begin{array}{l}{ }^{*} p<0.05 \\
{ }^{* *} p<0.01\end{array}$} \\
\hline immune score & $* * *$ & $\star * *$ & ${ }^{* *} p<0.01$ & immune score & 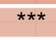 & & \\
\hline T cell regulatory (Tregs) & & & ${ }^{* * *} p<0.001$ & T cell regulatory (Tregs) & $* *$ & & ${ }^{* * *} p<0.001$ \\
\hline T cell gamma delta & & & Correlation & $T$ cell gamma delta & & & Correlation \\
\hline T cell NK & *** & *** & - & T cell NK & & *** & (a) \\
\hline$T$ cell $C D 8+$ naive & & & 0.25 & T cell $C D 8+$ naive & ** & *** & 0.2 \\
\hline T cell CD8+ effector memory & & & 0.00 & T cell CD8+ effector memory & & & 0.0 \\
\hline$T$ cell $C D 8+$ central memory & ${ }^{\star *}$ & *** & -0.25 & T cell CD8+ central memory & * & * & \\
\hline T cell CD8+ & ** & *** & -0.50 & T cell CD8+ & * & & -0.2 \\
\hline T cell CD4+ naive & * & * & & $T$ cell CD4+ naive & $\star * *$ & & \\
\hline$T$ cell CD4+ memory & & * & & $\mathrm{T}$ cell CD4+ memory & ** & & \\
\hline$T$ cell $C D 4+$ effector memory & $* * *$ & $* * *$ & & $T$ cell CD4+ effector memory & $* * *$ & & \\
\hline T cell CD4+ central memory & *** & $* *$ & & T cell CD4+ central memory & ** & * & \\
\hline T cell CD4+ Th2 & *** & $* * *$ & & T cell CD4+ Th2 & $* * *$ & $* * *$ & \\
\hline T cell CD4+ Th1 & *** & $* * *$ & & T cell CD4+ Th1 & $* * *$ & **** & \\
\hline T cell CD4+ (non-regulatory) & & & & T cell CD4+ (non-regulatory) & *** & & \\
\hline Plasmacytoid dendritic cell & & *** & & Plasmacytoid dendritic cell & & ** & \\
\hline Neutrophil & & $\star * *$ & & Neutrophil & & * & \\
\hline NK cell & & & & NK cell & & & \\
\hline Myeloid dendritic cell activated & $* * *$ & $* * *$ & & Myeloid dendritic cell activated & $* * *$ & & \\
\hline Myeloid dendritic cell & $\star \star * \star$ & 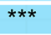 & & Myeloid dendritic cell & *** & & \\
\hline Monocyte & *** & *** & & Monocyte & *** & & \\
\hline Mast cell & 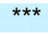 & & & Mast cell & $\star \star \star \star ~$ & * & \\
\hline Macrophage M2 & *** & *** & & Macrophage M2 & ** & & \\
\hline Macrophage M1 & & *** & & Macrophage M1 & & & \\
\hline Macrophage & ** & *** & & Macrophage & ** & & \\
\hline Hematopoietic stem cell & *** & $\star \star * *$ & & Hematopoietic stem cell & 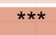 & & \\
\hline Granulocyte-monocyte progenitor & *** & *** & & Granulocyte-monocyte progenitor & $* * *$ & & \\
\hline Eosinophil & $\star \star * *$ & $\star \star \star$ & & Eosinophil & ** & & \\
\hline Endothelial cell & $* * *$ & $* * *$ & & Endothelial cell & $* * *$ & & \\
\hline Common myeloid progenitor & $* * *$ & 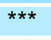 & & Common myeloid progenitor & * & & \\
\hline Common Iymphoid progenitor & *** & *** & & Common lymphoid progenitor & *** & * & \\
\hline Class-switched memory B cell & $* \star *$ & $\star *$ & & Class-switched memory B cell & $\star * \star$ & & \\
\hline B cell plasma & & $* * *$ & & B cell plasma & & ** & \\
\hline $\mathrm{B}$ cell naive & & & & B cell naive & $\star \star$ & * & \\
\hline B cell memory & ** & $* * *$ & & B cell memory & $* *$ & & \\
\hline B cell & * & $\star \star *$ & & $B$ cell & $\star \star \star \star$ & & \\
\hline & $v^{30}$ & $v^{5^{\circ}}$ & & & $v^{30}$ & $5^{50}$ & \\
\hline
\end{tabular}

levels in 22 lung cancer cell lines and 88 lung cancer samples, suggesting the potential of TIMELESS in diagnosis and prognosis of lung cancer [24]. The upregulation of TIMELESS has also been found in cervical carcinoma [25], colorectal cancer [26], and breast cancer [27], and the downregulation of RORA has been found in many cancers including hepatocellular carcinoma [28], gastric cancer [29], melanoma [30], and breast cancer [31]. In present study, we found that TIMELESS and RORA were two prognostic biomarkers in NSCLC. TIMELESS had tumor-promoting effects, and RORA had tumor-suppressing effects.

Accumulating evidence has shown that as a systemic disease, cancer involves the disorder of pathways and homeostasis in many aspects [32, 33]. Therefore, it is necessary to probe the role TIMELESS and RORA play in NSCLC systematically [34]. To further explore their functions, we identified genes that were significantly correlated with TIMELESS and RORA and found that genes positively correlated with TIMELESS expression were dramatically enriched in pathways related to the cell cycle and DNA replication. TIMELESS might interact with the eukaryotic elongation factor 1A2, which plays a role in cell migration and ribosomal protein biosynthesis [35]. Depletion of TIMELESS results in dysfunction of mitotic progression, impaired sister chromatid cohesion, and chromosome fragmentation [36]. Also, overexpression of claspin and TIMELESS can stabilize the replisome to reduce the chronic stress of replication on forks [37]. These data indicate the tumor-promoting effect of TIMELESS by promoting the cell cycle and DNA replication.

Interestingly, the significantly negatively correlated genes with TIMELESS were mainly enriched in the clusters strongly related to the immune system, antigen 


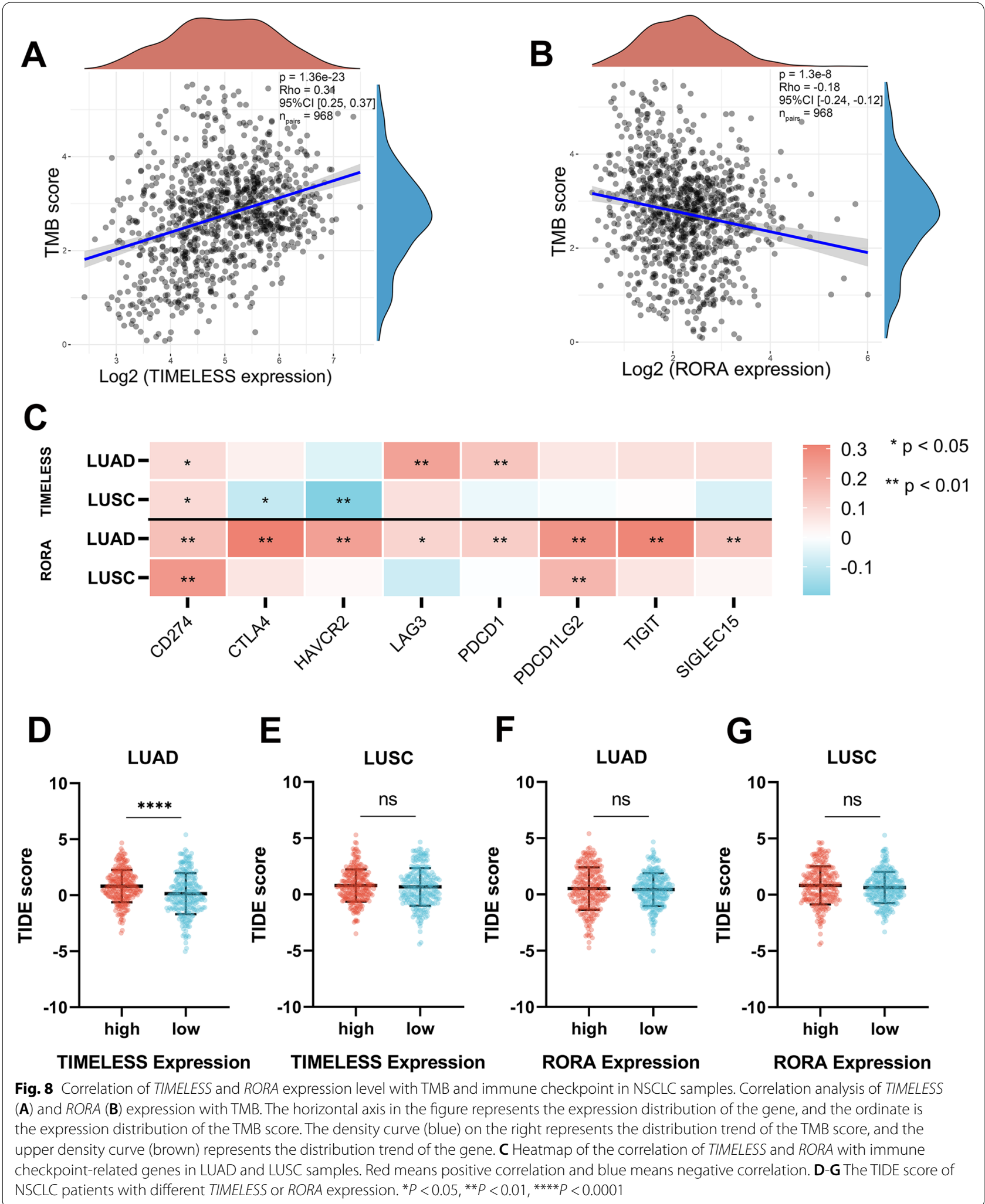




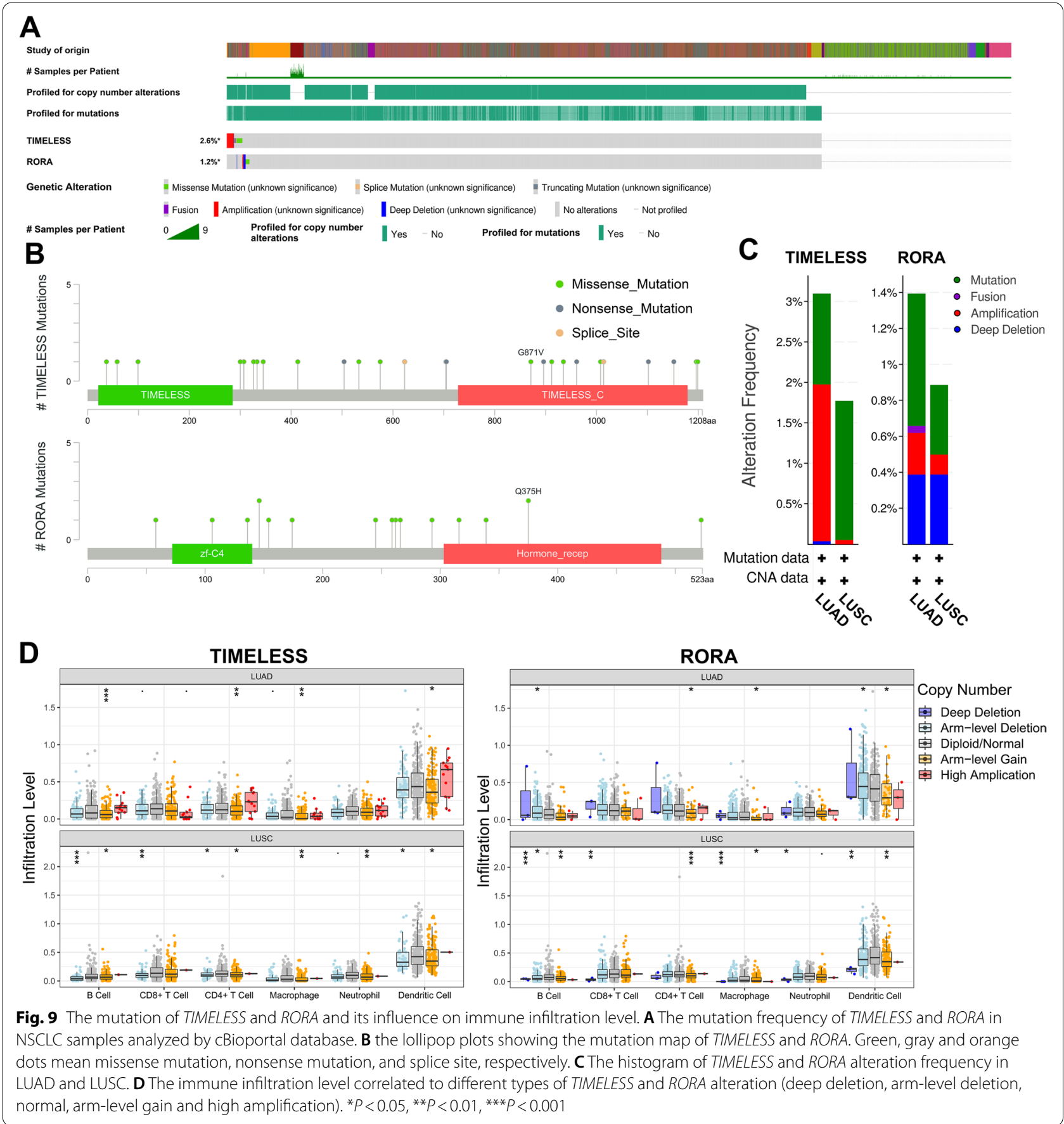

processing and presentation, complement and coagulation cascades, and rheumatoid arthritis. This suggests TIMELESS may take part in tumor immunological processes and exert inhibitory effects on tumor immunity. Therefore, we also discussed the relationship between TIMELESS and tumor immune function.

As an emerging independent predictor of sentinel lymph node status and tumor metastasis, tumor infiltrating lymphocytes has attracted considerable attention [38, 39]. So, we examined the potential correlation of TIMELESS and RORA expression with the immune infiltration levels in NSCLC by multiple algorithms (TIMER, "immuneeconv" $\mathrm{R}$ package, and XCELL). The negative correlation between TIMELESS expression and infiltration levels of most immune cells was observed according to TIMER and $R$ package analysis, while the XCELL 
algorithm indicated a significant positive correlation of TIMELESS expression level and CD4+ Th1, Th2 cells. And RORA expression was positively correlated with most immune cell infiltration especially in LUAD. The immune infiltration levels of different algorithms were broadly in line with each other, especially for TIMELESS in LUSC and RORA in LUAD. These results were broadly consistent with functional enrichment analyses, indicating the importance of TIMELESS and RORA in the recruitment and regulation of immune infiltrating cells in NSCLC.

As an innovative therapy, considerable attention has been paid to immunotherapy in cancer treatment. Immune checkpoint inhibitors (mainly $P D-1 / P D-L 1$ targets) have shown considerable advantages for several tumor types, especially NSCLC [40]. Therefore, it is of great significance to evaluate the effects of TIMELESS and RORA on ICBs. A high TMB is likely to increase the capacity of a tumor to generate neoantigens. The increased productions of neoantigens would be expected to render a tumor more immunogenic, thus increasing its likelihood of responding to immunotherapy. TMB may be characterized concerning illustration the aggregate number for physical mutations for every megabase or those nonsynonymous transformations in tumor tissues, including supplanting and insertion erasure changes, and has become a preferable biomarker to foresee the light of anti-PD-1/PD-L1 therapy [41, 42]. Higher TMB is associated with higher immunogenicity and response to ICBs in lung cancer [43], and some meta-analyses also indicated a strong positive correlation [42, 44]. Interestingly, our results illustrated that there were significant correlations of TMB score with TIMELESS (positive) and RORA (negative) expression, indicating that TIME$L E S S$ and RORA expression level could estimate the $P D-1 / P D-L 1$ blockers therapy response of patients as biological markers in some degree. We also found that TIMELESS was significantly positively related to CD274, $L A G 3$, and PDCD1 in LUAD as well as CD274 in LUSC, and negatively related to CTLA4 and HAVCR2 in LUSC. $R O R A$ was significantly positively correlated with all immune checkpoint-related genes in LUAD and CD274 and PDCD1LG2 in LUSC. The tumor immune dysfunction and exclusion (TIDE) algorithm was mainly utilized to predict ICB response in NSCLC and melanoma, in which multiple gene expression markers were used to estimate the dysfunction and exclusion of tumor infiltration cytotoxic T lymphocytes (CTLs) [21]. In this study, LUAD patients with high TIMELESS expression had a significantly higher TMB score than those with low TIMELESS expression, indicating a better response to ICB therapy and more benefits. Collectively, these results indicated the probable potential of TIMELESS and RORA as predictors on the response to ICB therapy. However, as the therapy details of the data we used in those databases was not so clear, there are no direct clinical data to confirm the prediction effect of TIMELESS and RORA. The specific prediction effects of them, as well as the correlation of those factors require much further clinical exploration.

Tumor lipid metabolism has attracted extensive attention recently. Aberrant lipid metabolism is found in multiple human cancers [45], and lipid synthesis is essential for neoplastic growth in many cancer types [46]. More importantly, some molecules in lipogenesis have been identified to have tumor-promoting roles such as tumor invasion, metastasis, and other malignant biologic function. For example, fatty acid synthase (FASN) was upregulated in multiple cancer types [47] and is correlated with the amplification of HER2 in breast cancer [48]; also, ATP-Citrate Lyase $(A C L Y)$ was upregulated in gastric adenocarcinoma [49], and downregulation of $A C L Y$ could decrease cell proliferation and invasion in multiple cancer cells [50]. We specifically examined the correlation of TIMELESS and RORA with lipid metabolism. The results indicated that molecules in lipid metabolism including $S C D, F A S N, A C A C A, A C L Y, H M G C S 1, H M G C R, H I F 1 A$, $F A D S 1, F A D S 2, C P T 1 A$, and CHPT1 were strongly positively correlated with TIMELESS. TIMELESS upregulated $A C E R 2$ expression and mitochondrial respiration thus increased S1P synthesis in ER-positive breast cancer and is closely correlated with poor prognosis [51].

In addition, the immune cells in tumor microenvironment also have abnormal lipid metabolism and cause abnormal tumor immunity. Nevertheless, there is a correlation between abnormal lipid metabolism and tumor immunity. TIMELESS maybe the key gene related to tumor and immune and lipid metabolism. Or it is possible to find an effective recognition of the metabolic pathways shared between tumor and immune cells through TIMELESS, therefore targeting tumor metabolism and reprogramming immune cell metabolism.

\section{Conclusion}

Collectively, we identified two genes, TIMELESS and $R O R A$, as the key clock genes in NSCLC. Their importance in the pathogenesis of NSCLC was proved from the perspective of prognosis and gene expression. Moreover, using bioinformatics analysis, we probed the function of TIMELESS and RORA in multiple aspects, especially in tumor immune regulation and TIMELESS in lipid metabolism. These results indicate the strong potential of TIMELESS and RORA to be the biomarkers for NSCLC progression and prognosis. 


\begin{abstract}
Abbreviations
NSCLC: Non-small cell lung cancer; PER1: Period circadian regulator 1; PER2: Period circadian regulator 2; CRY1: Cryptochrome circadian regulator 1; CRY2: Cryptochrome circadian regulator 2; TIMELESS: Timeless circadian regulator; RORA: Retinoic acid-related orphan receptor A; NR1D1: Nuclear receptor subfamily 1, group D member 1; NR1D2: Nuclear receptor subfamily 1, group D member 2; TNM: Tumor-node-metastasis; LUAD: Lung adenocarcinoma; LUSC: Lung squamous cell carcinoma; TCGA: The Cancer Genome Atlas; GTEx: Genotype-Tissue Expression; GO: Gene Ontology; KEGG: Kyoto Encyclopedia of Genes and Genomes; TMB: Tumor mutation burden; TIDE: Tumor immune dysfunction and exclusion; ICB: Immune checkpoint blockade; CTLs: Cytotoxic Tlymphocytes; TIMER: Tumor immune estimation resource; mDCs: Myeloid dendritic cells; OS: Overall survival; PFS: Progression-free survival; PPS: Postprogression survival; FASN: Fatty acid synthase; ACLY: ATP-Citrate Lyase.
\end{abstract}

\section{Supplementary Information}

The online version contains supplementary material available at https://doi. org/10.1186/s12885-022-09203-1.

Additional file 1: Table S1. Genes significantly related to TIMELESS and RORA.

\section{Acknowledgements}

Not applicable.

\section{Authors' contributions}

HX completed most of the bioinformatics analysis and wrote the manuscript $Y L, B Z, Y C, H Y$, and $X L$ provided insights, completed the expression and survival analysis, and revised the manuscript. YP designed the research and wrote the manuscript. All authors read and approved the final manuscript.

\section{Funding}

National Natural Science Foundation of China $(81773765 ; 81270049$; 30901815; 81874318; 81673453; 81473235) and Applied Basic Research Program of Shanxi Province (201901D211470) funded project. The funders did not play a role in the design of the study and collection, analysis, and interpretation of data or in writing the manuscript.

\section{Availability of data and materials}

The data of the current study is available from the following open public databases: TCGA (https://cancergenome.nih.gov), Oncomine (https://www. oncomine.org/), and GTEx (https://gtexportal.org/home/datasets) as is described above. Other data will be available from the corresponding author upon reasonable request.

\section{Declarations}

\section{Ethics approval and consent to participate}

The authors are accountable for all aspects of the work in ensuring that questions related to the accuracy or integrity of any part of the work are appropriately investigated and resolved. The trial was conducted in accordance with the Declaration of Helsinki (as revised in 2013). The collection of tissue samples was approved by ethics committees of the Third Affiliated Hospital of Kunming Medical University (No: KYCS202180), and written informed consent was obtained from all patients before the tissue sample collection.

\section{Consent for publication}

Not applicable.

\section{Competing interests}

The authors declared no competing interests.

\section{Author details}

'Department of Pharmacology, School of Basic Medical Sciences, Health Science Center, Peking University, Beijing 100191, China. ${ }^{2}$ Department of Rehabilitation Medicine, Kunming Medical University, 1168 Western Chunrong Road, Yuhua Street, Chenggong District, Kunming 650500, Yunnan,
China. ${ }^{3}$ Beijing Key Laboratory of Tumor Systems Biology, Peking University, Beijing 100191, China.

Received: 11 October 2021 Accepted: 13 January 2022

Published online: 25 January 2022

\section{References}

1. Siegel RL, Miller KD, Jemal A. Cancer statistics, 2016. CA Cancer J Clin. 2016;66(1):7-30.

2. Chen Z, Fillmore CM, Hammerman PS, Kim CF, Wong K-K. Non-smallcell lung cancers: a heterogeneous set of diseases. Nat Rev Cancer. 2014;14(8):535-46.

3. Huang WT, Cen WL, He RQ, Xie Y, Zhang Y, Li P, et al. Effect of miR-146a-5p on tumor growth in NSCLC using chick chorioallantoic membrane assay and bioinformatics investigation. Mol Med Rep. 2017;16(6):8781-92.

4. Halliday PR, Blakely CM, Bivona TG. Emerging targeted therapies for the treatment of non-small cell lung cancer. Curr Oncol Rep. 2019;21(3):1-12.

5. Mogavero F, Jager A, Glennon JC. Clock genes, ADHD and aggression. Neurosci Biobehav Rev. 2018;91:51-68.

6. Cox KH, Takahashi JS. Circadian clock genes and the transcriptional architecture of the clock mechanism. J Mol Endocrinol. 2019;63(4):R93-R102.

7. Straif K, Baan R, Grosse Y, Secretan B, El Ghissassi F, Bouvard V, Altieri A, Benbrahim-Tallaa L, Cogliano V, Group WIAfRoCMW: Carcinogenicity of shift-work, painting, and fire-fighting. 2007.

8. Salamanca-Fernández E, Rodríguez-Barranco M, Guevara M, Ardanaz E, Sánchez M. Night-shift work and breast and prostate cancer risk: updating the evidence from epidemiological studies. Anales del sistema sanitario de Navarra. 2018;41(2):211-26.

9. Papantoniou K, Devore EE, Massa J, Strohmaier S, Vetter C, Yang L, et al. Rotating night shift work and colorectal cancer risk in the nurses' health studies. Int J Cancer. 2018;143(11):2709-17.

10. Couto P, Miranda D, Vieira R, Vilhena A, De Marco L, Bastos-Rodrigues L. Association between CLOCK, PER3 and CCRN4L with non-small cell lung cancer in Brazilian patients. Mol Med Rep. 2014;10(1):435-40.

11. Filipski E, Lévi F. Circadian disruption in experimental Cancer processes. Integr Cancer Ther. 2009;8(4):298-302.

12. Filipski E, King VM, Li X, Granda TG, Mormont M-C, Claustrat B, et al. Disruption of circadian coordination accelerates malignant growth in mice. Pathol Biol. 2003;51(4):216-9.

13. Lee S, Donehower LA, Herron AJ, Moore DD, Fu L. Disrupting circadian homeostasis of sympathetic signaling promotes tumor development in mice. PLoS One. 2010;5(6):e10995.

14. Papagiannakopoulos T, Bauer MR, Davidson SM, Heimann M, Subbaraj L, Bhutkar A, et al. Circadian rhythm disruption promotes lung tumorigenesis. Cell Metab. 2016;24(2):324-31.

15. Mteyrek A, Filipski E, Guettier C, Okyar A, Lévi F. Clock gene Per2 as a controller of liver carcinogenesis. Oncotarget. 2016;7(52):85832.

16. Rhodes DR, Kalyana-Sundaram S, Mahavisno V, Varambally R, Yu J, Briggs BB, et al. Oncomine 3.0: genes, pathways, and networks in a collection of 18,000 cancer gene expression profiles. Neoplasia. 2007;9(2):166-80.

17. Győrffy B, Surowiak P, Budczies J, Lánczky A. Online survival analysis software to assess the prognostic value of biomarkers using transcriptomic data in non-small-cell lung cancer. PLoS One. 2013;8(12):e82241.

18. Livak KJ, Schmittgen TD. Analysis of relative gene expression data using real-time quantitative PCR and the $2-\triangle \triangle C T$ method. methods. 2001;25(4):402-8.

19. Kanehisa M, Furumichi M, Sato Y, Ishiguro-Watanabe M, Tanabe M KEGG: integrating viruses and cellular organisms. Nucleic Acids Res. 2021;49(D1):D545-d551.

20. You Z-P, Zhang Y-L, Li B-Y, Zhu X-G, Shi K. Bioinformatics analysis of weighted genes in diabetic retinopathy. Invest Ophthalmol Vis Sci. 2018;59(13):5558-63.

21. Jiang P, Gu S, Pan D, Fu J, Sahu A, Hu X, et al. Signatures of T cell dysfunction and exclusion predict cancer immunotherapy response. Nat Med. 2018;24(10):1550-8.

22. Li T, Fu J, Zeng Z, Cohen D, Li J, Chen Q, et al. TIMER2. 0 for analysis of tumor-infiltrating immune cells. Nucleic Acids Res. 2020;48(W1):W509-14. 
23. Gao J, Aksoy BA, Dogrusoz U, Dresdner G, Gross B, Sumer SO, et al. Integrative analysis of complex cancer genomics and clinical profiles using the cBioPortal. Sci Signal. 2013;6(269):pl1.

24. Yoshida K, Sato M, Hase T, Elshazley M, Yamashita R, Usami N, et al. TIMELESS is overexpressed in lung cancer and its expression correlates with poor patient survival. Cancer Sci. 2013;104(2):171-7.

25. Zhang W, He W, Shi Y, Zhao J, Liu S, Zhang F, et al. Aberrant TIMELESS expression is associated with poor clinical survival and lymph node metastasis in early-stage cervical carcinoma. Int J Oncol. 2017;50(1):173-84.

26. Mazzoccoli G, Panza A, Valvano M, Palumbo O, Carella M, Pazienza V, et al. Clock gene expression levels and relationship with clinical and pathological features in colorectal cancer patients. Chronobiol Int. 2011;28(10):841-51.

27. Nishikimi A, Uruno T, Duan X, Cao Q, Okamura Y, Saitoh T, et al. Blockade of inflammatory responses by a small-molecule inhibitor of the Rac activator DOCK2. Chem Biol. 2012;19(4):488-97.

28. Fu R-D, Qiu C-H, Chen H-A, Zhang Z-G, Lu M-Q. Retinoic acid receptorrelated receptor alpha (RORalpha) is a prognostic marker for hepatocellular carcinoma. Tumor Biol. 2014;35(8):7603-10.

29. Wang Z, Xiong F, Wang X, Qi Y, Yu H, Zhu Y, et al. Nuclear receptor retinoid-related orphan receptor alpha promotes apoptosis but is reduced in human gastric cancer. Oncotarget. 2017;8(7):11105-13.

30. Li B, Wang Y, Xu Y, Liu H, Bloomer W, Zhu D, et al. Genetic variants in RORA and DNMT1 associated with cutaneous melanoma survival. Int J Cancer. 2018;142(11):2303-12.

31. Du J, Xu R. RORa, a potential tumor suppressor and therapeutic target of breast cancer. Int J Mol Sci. 2012;13(12):15755-66.

32. Hornberg JJ, Bruggeman FJ, Westerhoff HV, Lankelma J. Cancer: a systems biology disease. Biosystems. 2006;83(2-3):81-90.

33. Masoudi-Nejad A, Bidkhori G, Ashtiani SH, Najafi A, Bozorgmehr JH, Wang E. Cancer systems biology and modeling: microscopic scale and multiscale approaches. In: Seminars in cancer biology: 2015: Elsevier; 2015. p. 60-9.

34. Werner HM, Mills GB, Ram PT. Cancer systems biology: a peek into the future of patient care? Nat Rev Clin Oncol. 2014;11(3):167.

35. Gotter AL, Suppa C, Emanuel BS. Mammalian TIMELESS and Tipin are evolutionarily conserved replication fork-associated factors. J Mol Biol. 2007;366(1):36-52.

36. Leman AR, Noguchi C, Lee CY, Noguchi E. Human Timeless and Tipin stabilize replication forks and facilitate sister-chromatid cohesion. J Cell Sci. 2010;123(5):660-70.

37. Bianco JN, Bergoglio V, Lin Y-L, Pillaire M-J, Schmitz A-L, Gilhodes J, et al. Overexpression of Claspin and Timeless protects cancer cells from replication stress in a checkpoint-independent manner. Nat Commun. 2019;10(1):910.

38. Azimi F, Scolyer RA, Rumcheva P, Moncrieff M, Murali R, McCarthy SW, et al. Tumor-infiltrating lymphocyte grade is an independent predictor of sentinel lymph node status and survival in patients with cutaneous melanoma. J Clin Oncol. 2012;30(21):2678-83.

39. Pan J-h, Zhou H, Cooper L, J-I H, Zhu S-b, X-x Z, et al. LAYN Is a Prognostic Biomarker and Correlated With Immune Infiltrates in Gastric and Colon Cancers. Front Immunol. 2019;10:6.

40. Herzberg B, Campo MJ, Gainor JF. Immune checkpoint inhibitors in nonsmall cell lung Cancer. Oncologist. 2017;22(1):81-8.

41. Chae YK, Davis AA, Agte S, Pan A, Simon NI, lams WT, et al. Clinical implications of circulating tumor DNA tumor mutational burden (ctDNA TMB) in non-small cell lung Cancer. Oncologist. 2019;24(6):820-8.

42. Zhu J, Zhang T, Li J, Lin J, Liang W, Huang W, et al. Association between tumor mutation burden (TMB) and outcomes of Cancer patients treated with PD-1/PD-L1 inhibitions: a Meta-analysis. Front Pharmacol. 2019;10:673.

43. Goodman AM, Kato S, Bazhenova L, Patel SP, Frampton GM, Miller $\checkmark$, et al. Tumor mutational burden as an independent predictor of response to immunotherapy in diverse cancers. Mol Cancer Ther. 2017;16(11):2598-608.

44. Osipov A, Lim SJ, Popovic A, Azad NS, Laheru DA, Zheng L, et al. Tumor mutational burden, toxicity, and response of immune checkpoint inhibitors targeting PD(L)1, CTLA-4, and combination: a Meta-regression analysis. Clin Cancer Res. 2020;26(18):4842-51.
45. Peck B, Schulze A. Lipid metabolism at the Nexus of diet and tumor microenvironment. Trends in Cancer. 2019;5(11):693-703.

46. Gebhard R, Clayman R, Prigge W, Figenshau R, Staley N, Reesey C, et al. Abnormal cholesterol metabolism in renal clear cell carcinoma. J Lipid Res. 1987;28(10):1177-84.

47. Menendez JA, Lupu R. Fatty acid synthase and the lipogenic phenotype in cancer pathogenesis. Nat Rev Cancer. 2007;7(10):763-77.

48. Kumar-Sinha C, Ignatoski KW, Lippman ME, Ethier SP, Chinnaiyan AM. Transcriptome analysis of HER2 reveals a molecular connection to fatty acid synthesis. Cancer Res. 2003;63(1):132-9.

49. Qian X, Hu J, Zhao J, Chen H. ATP citrate lyase expression is associated with advanced stage and prognosis in gastric adenocarcinoma. Int J Clin Exp Med. 2015;8(5):7855.

50. Xin M, Qiao Z, Li J, Liu J, Song S, Zhao X, et al. miR-22 inhibits tumor growth and metastasis by targeting ATP citrate lyase: evidence in osteosarcoma, prostate cancer, cervical cancer and lung cancer. Oncotarget. 2016;7(28):44252.

51. Zhang S, Huang P, Dai H, Li Q, Hu L, Peng J, et al. TIMELESS regulates sphingolipid metabolism and tumor cell growth through Sp1/ACER2/S1P axis in ER-positive breast cancer. Cell Death Dis. 2020;11(10):1-14.

\section{Publisher's Note}

Springer Nature remains neutral with regard to jurisdictional claims in published maps and institutional affiliations.

Ready to submit your research? Choose BMC and benefit from:

- fast, convenient online submission

- thorough peer review by experienced researchers in your field

- rapid publication on acceptance

- support for research data, including large and complex data types

- gold Open Access which fosters wider collaboration and increased citations

- maximum visibility for your research: over $100 \mathrm{M}$ website views per year

At BMC, research is always in progress.

Learn more biomedcentral.com/submissions 